\title{
A Redundant Parallel Manipulator with Constrained Chains
}

\author{
Dongdong Wang ${ }^{\mathrm{a},}{ }^{*}$, Xiankui Zhang $^{\mathrm{b}}$ and Biao Hou ${ }^{\mathrm{c}}$ \\ IEST, Harbin Institute of Technology, Harbin 150000, China \\ a2812471443@qq.com, bzhangxk@hit.edu.cn, c982721053@qq.com
}

Keywords: parallel manipulator, pseudo-inverse, optimization, inverse dynamics.

\begin{abstract}
This paper has designed and analyzed a kind of parallel manipulator with redundant actuators. The manipulator has different DOFs and motions comparing to conventional parallel manipulators, which is mainly used to simulate various position of heavy, large equipments (such as buses, trucks), due to the position of three constrained chains. In this paper, the conceive outline and a clear structure of this mechanism has been presented at first. By building up an effective kinematic model, the movements of the output platform are analyzed. In addition, the constraint equations are obtained in order to estimate the relationship between self-movement and driven motion. Furthermore, the kinematic and dynamic characteristics have been investigated with common method. Finally, for the redundant force, an optimal approach correlated with output direction is proposed to acquire better-proportioned results than other approaches.
\end{abstract}

\section{Introduction}

As a kind of complicated mechanism, parallel manipulator has been widely used in various fields since invented in terms of its load-carrying capacity, accuracy and rigidity in comparison to corresponding serial ones [13]. W.L.G. Pollard published his patent describing an auto parallel device used as a spray painting machinery [1]. Eric Gough invented a parallel machine to test tires [2]. Recently, parallel manipulator has made new progress in several areas such as machine tools, precision control, manufacturing in particular the system simulation $[4,5,6,10]$.

Over the past decades, lots of researchers focused on the structure of parallel mechanism and put forward a series of theories. The very first paper was delivered by Stewart [3], which described a 6DOF parallel manipulator and analyzed the kinematics. The appearance of Delta [4] struck the industry world with amazement. The Delta robot made new breakthrough in the quick pick-and-place operations. Shortly after its application, François Pierrot et al. altered traveling plate into a new structure named PAR4 [5], which has 4-DOF. A. Hernandez presented translational parallel manipulator with $\mathrm{Pa}^{2}$ kinematic joints [8]. Kunhai Cai et al. designed a 6-DOF parallel appliance combined with two 3-DOF stages [10]. Along with many variations of the parallel manipulators, the theories also have developed. Hunt [9] drew screw theory into parallel machines at the first time. Huang applied the theory successfully [7]. However, there is none universal method for the kinetic and dynamic problems. For a new manipulator, its characteristics should be studied and verified comprehensively.

Owning to the good performance of rigidity and load-carrying capacity, a kind of parallel manipulator (see Fig.1) has been chosen as the device to simulate working conditions of heavy equipments in this paper. In order to improve the overall stiffness again, the manipulator has increased three constrained chains and redundant actuator.

In [15], we know that kinematically redundant manipulators not only have significant improvement in the force capabilities but also can avoid singularity. In the resolution of redundancy, most optimization methods are based on the 2-norm and $\infty$-norm. Yuan F. Zheng and J.Y.S. Luh [11] compared two optimal algorithms: least energy consumption and load distribution with minimum exerted forces. They found that the second method could get less computational time. YOSHIHIKO NAKAMURA [12] aimed at solving the redundant problem of the inverse dynamics of closed-link mechanisms. He transformed the optimization of actuation redundancy into a typical Quadratic Programming problem. 


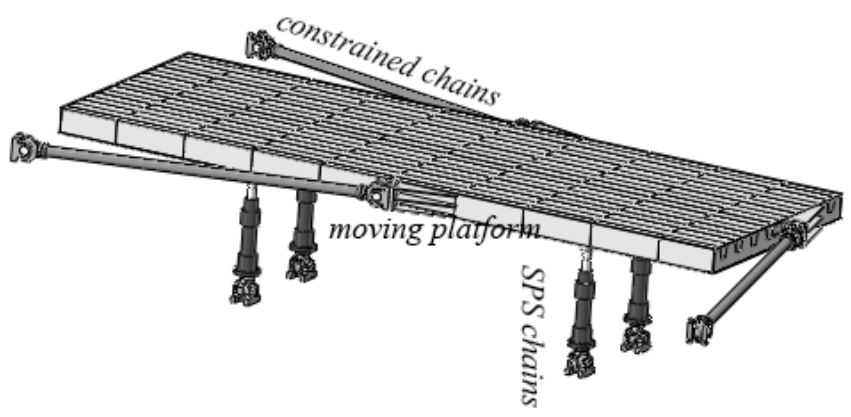

Fig. 1 The parallel manipulator without the base

And he concluded that Closed-link mechanism with actuation redundancy have advantages for increasing the payload and improving the dynamic response. Mats Isaksson et al. [13] proposed two novel fault tolerance indices applied to a 3-DOF planar parallel mechanism. Sung-Hoon Cha et al. [14] designed a cost function based on local optimization to avoid the most problematic singularity configurations. Travis Baratcart et al. [15] introduced the cCGI (Continuous Cascaded Generalized Inverse) algorithm which can guarantee continuity in resolution and expand the output range. S.B. Nokleby et al. [16] presented a methodology to calculate the force capabilities determined by scaling factors correlated with actuator limits of non-redundantly and redundantly-actuated parallel manipulators.

In this paper, a method reflecting the distribution of all actuators' force based on predominant force direction has been introduced. This method use coefficient associated with corresponding angles and force limits to determine the allocation proportion. An optimation approach on basis of this method is derived to calculate redundant forces. This method could get a better-proportioned and continuous result.

This paper presents the structure of the parallel manipulator and analyses the motion at first. Next, the constrained equations are solved to attest the relationship between self-movement and transport motion. Then, according to the result of these equations, the kinematic and dynamic characteristics are derived. Finally, the coefficient optimation approach is applied to solve the redundant force.

\section{The Parallel Manipulator}

\subsection{The Mechanical Structure}

As shown in Fig.1, the manipulator is consisted of a basement and a moving platform linked by seven legs $A_{i} B_{i}(i=1 \sim 7)$.Three constrained chains are parallel with ground as to be install conveniently, which make DOF numbers different with 4-SPS manipulators. The load is put on the moving platform. Along with the movements of traveling plate, the working conditions of load are simulated. Every SPS chain has an active actuator P (prismatic joint).

All the SPS chains propel traditional and rotational motions of the moving platform.

The schematic plot is shown in Fig.2.A fixed coordinate system O-XYZ is built at the center of the up-platform. $X$ axis and $Y$ axis are parallel with the two side of moving platform. $Z$ axis points to the ground perpendicular to XY plane. The moving reference frame P-uvw is located at the same position when in static condition. Other structure parameters can be seen in Fig.2.

\subsection{Mobility}

The movements of parallel manipulators are a significant parameter, which determine the inputs when solving the inverse kinematics introduced later. So, the motion capability is the first issue to address. According to the Grübler-Kutzbach formula:

$$
\mathrm{M}=6(\mathrm{n}-\mathrm{g}-1)+\sum_{i=1}^{g} f_{i}
$$




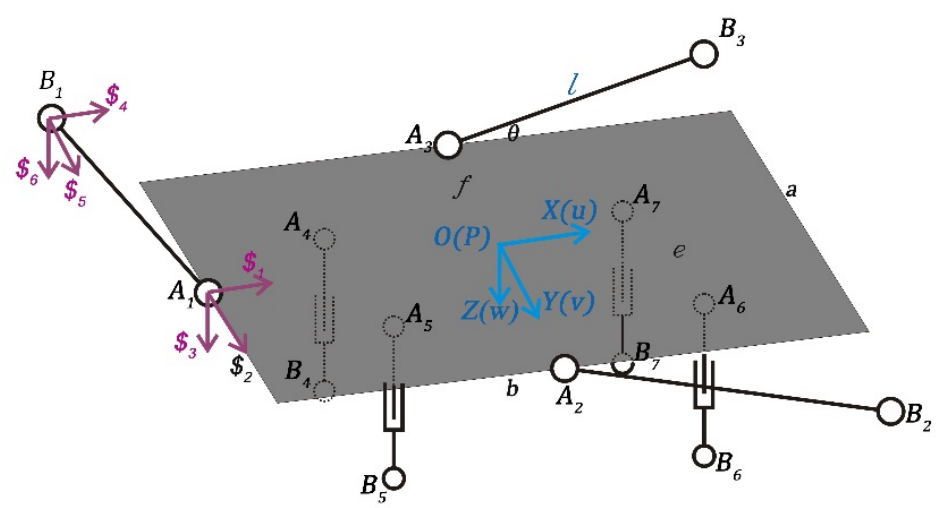

Fig.2 The schematic plot

Where $\mathrm{n}$ is the number of components, $\mathrm{g}$ is the number of joints, $f_{i}$ is the DOF number of $i$ th joint. In this parallel manipulator: $n=13, g=18$, total DOF number is 46 . However, there exists rotations around the axes as local degree of freedom in SPS legs. Hence, the whole mechanism has 3 DOF.

Huang Zhen analyzed DOF of most parallel manipulators in detail successfully by reciprocal screw theory [7]. We also use this method to obtain specific freedom. Four SPS chains do not confine any movements in the manipulator. Three constrained chains would limit three motions in different directions. Take $A_{1} B_{1}$ as example, the motion screws can be expressed as:

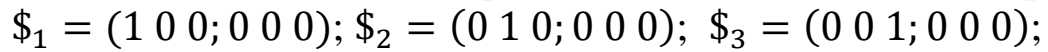

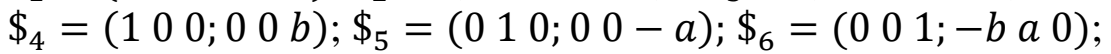

The reciprocal screw can be calculated as:

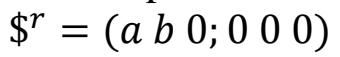

It implies that $A_{1} B_{1}$ causes a constraint force along the direction of the link axis to the moving platform. Meanwhile, the other two constrained chains form respective forces alongprep $A_{i} B_{i}(i=$ 2or3).As we know from the reciprocal screw theory, the three unintersectant forces in one plane would limit any movement in the plane and the rotation of the normal direction to the plane. Therefore, the three DOF includes two rotational DOF around X, Y axis and a traditional DOF along $\mathrm{Z}$ axis.

\subsection{Convected Motion}

The parallel manipulator which has DOF number less than six always exists convected motion. This kind of motion connects self-motion DOF with driven motion DOF together. In this paper, although three DOF have been analyzed formerly, indeed the other three DOF also exist as a result of the convected motion. Three constrained legs lead to three constraint equations:

$\left|\boldsymbol{A}_{i}^{\prime}-\boldsymbol{B}_{i}^{\prime}\right|=l \quad i=1,2,3$

Where $\boldsymbol{A}_{i}^{\prime}, \boldsymbol{B}_{i}^{\prime}$ represent spherical joints' coordinates of the constrained legs in the moving reference frame.

\subsection{Novelty}

Due to the existence of the convected motion, the table can implement the precise location by the simultaneously motions in two directions. The constrained legs increase the rigidity to sustain the heavy, large loads.

\section{Inverse Kinematics Analysis}

The inverse kinematics problem studies the mapping relationship from the outputs to the active joint inputs. The mapping is defined as a kind of coordinate transformation between the fixed reference frame and moving coordinate system. Euler angles are used to describe the gestures of upplatform. The position vector of the platform can be expressed as:

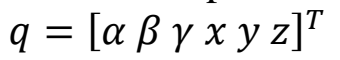

$\alpha, \beta, \gamma$ are denoted as the Euler angles, $x, y, z$ is the location of point P. According to the above, $\alpha, \beta, z$ are known. $\gamma, x, y$ should be calculated by the constraint equations. The coordinate of joint point $A_{i}, B_{i}$ in the reference O-XYZ can be written as: 


$$
\begin{aligned}
& A=\left[A_{1}, \ldots, A_{7}\right]=\left[\begin{array}{ccccccc}
-0.5 b & 0 & 0 & -0.5 f & -0.5 f & 0.5 f & 0.5 f \\
0 & 0.5 a & -0.5 a & -0.5 e & 0.5 e & 0.5 e & -0.5 e \\
0 & 0 & 0 & 0 & 0 & 0 & 0
\end{array}\right] \\
& B=\left[B_{1}, \ldots, B_{7}\right]= \\
& \left.\begin{array}{ccccccc}
-(0.5 b+l \sin \theta) & l \cos \theta & l \cos \theta & -0.5 f & -0.5 f & 0.5 f & 0.5 f \\
-l \cos \theta & 0.5 a+l \sin \theta & -(0.5 a+l \sin \theta) & -0.5 e & 0.5 e & 0.5 e & -0.5 e \\
0 & 0 & 0 & d & d & d & d
\end{array}\right]
\end{aligned}
$$

The transition matrix from fixed reference frame to moving coordinate system can be noted as:

$$
\mathrm{T}=\left[\begin{array}{ccc}
\cos \beta \cos \gamma & -\cos \alpha \sin \gamma+\sin \alpha \sin \beta \cos \gamma & \sin \alpha \sin \gamma+\cos \alpha \sin \beta \cos \gamma \\
\cos \beta \sin \gamma & \cos \alpha \cos \gamma+\sin \alpha \sin \beta \sin \gamma & -\sin \alpha \cos \gamma+\cos \alpha \sin \beta \sin \gamma \\
-\sin \beta & \sin \alpha \cos \beta & \cos \alpha \cos \beta
\end{array}\right]
$$

Then Eq. 2 can be written by the form:

$\left|\mathrm{T} A_{i}-B_{i}+t\right|=l(i=1,2,3)$

Eq.3 are three functions with respect to variables $\gamma, x, y$ determined by given values $\alpha, \beta, z$. By means of calculating these functions, we can ensure the whole position $q$ of output platform. This step is necessary to solve the inverse kinematics problem. Next step is to find the mapping from the pose of reference point $\mathrm{P}$ in $\mathrm{O}-\mathrm{XYZ}$ to the set of actuated joint inputs. The displacements of the active prismatic joints can be expressed as:

$d_{i}=\left|\mathrm{T} A_{i}-B_{i}+t\right|-d \quad i=4,5,6,7$

Apart from the position analysis, it also involves mapping from the general output velocity to actuated input velocity. By the conventional method, the velocities of the actuators can be obtained as Fig.(5):

$\dot{d}=J_{l q}\left[\begin{array}{c}v \\ \omega\end{array}\right]$

$J_{l q}$ is the Jacobian matrix mapping from output velocities of moving platform to the input velocities of active actuators which is calculated as $\left[D_{n}^{T}\left(T A \times D_{n}\right)\right]^{T} \cdot\left[\begin{array}{l}v \\ \omega\end{array}\right]$ are the general velocities of moving platform. $\mathrm{D}_{n}$ indicates unit vector of the actuator direction.

As mentioned before, we only know $\dot{\alpha} 、 \dot{\beta} 、 \dot{z}$ in the general velocities. The calculation of the parameters $\dot{x} 、 \dot{y} 、 \dot{\gamma}$ should refer to the constraint equation Eq.(3):

$$
\left[\begin{array}{c}
\dot{x} \\
\dot{y} \\
\dot{\gamma}
\end{array}\right]=\left[\begin{array}{lll}
\frac{\partial x}{\partial \alpha} & \frac{\partial x}{\partial \beta} & \frac{\partial x}{\partial z} \\
\frac{\partial y}{\partial \alpha} & \frac{\partial y}{\partial \beta} & \frac{\partial y}{\partial z} \\
\frac{\partial \gamma}{\partial \alpha} & \frac{\partial \gamma}{\partial \beta} & \frac{\partial \gamma}{\partial z}
\end{array}\right]\left[\begin{array}{c}
\dot{\alpha} \\
\dot{\beta} \\
\dot{\gamma}
\end{array}\right]
$$

The coefficient matrix in Eq. (6) can be derived by the derivative of Eq. (3). Take the first column as the example:

Rewrite the constraint equation as:

$h_{i}=\left|\mathrm{T} A_{i}-B_{i}+t\right|-l \quad i=1,2,3$

establish simultaneous equations involving derivative:

$$
\left\{\begin{array}{l}
\frac{\partial h_{1}}{\partial \alpha}=0 \\
\frac{\partial h_{2}}{\partial \alpha}=0 \\
\frac{\partial h_{3}}{\partial \alpha}=0
\end{array}\right.
$$

Then the values of first column in the matrix can be achieved by solving Eq. (8).

\section{Inverse Dynamics}

The solution of the inverse kinematics and the convected motion laid the foundation to solve the inverse dynamics. The inverse dynamics problem is to find out forces of the active actuators which can achieve the general given pose of the output platform. The conventional methods to solve this problem includes Newton-Euler, Lagrange, Kane method and so on. In this paper, we can simplify 
the parallel manipulator as a single rigid body because of the much larger mass of load and platform than other components. On the basis of the assumption, the dynamic model can be analyzed by setting up Newton-Euler equations which can be written as:

$$
\left\{\begin{array}{c}
D_{n} F+Q=F_{g} \\
\left(T A \times D_{n}\right) F=M_{t}
\end{array}\right.
$$

where $\mathrm{F}$ is the unknow variable to be determined, denoted as the output forces matrix is the gravitational vector. $F_{g}$ is the inertia force about whole system with respect to general acceleration of moving plate, as shown in Eq. (10):

$$
F_{g}=m[\ddot{x} \quad \ddot{y} \quad \ddot{z}]^{T}
$$

The symbol in Euler equation $M_{t}$ indicates total inertia moment, which can be described as:

$M_{t}=I \dot{\omega}+\omega \times I \omega$

where I represents inertia matrix of the output platform in moving reference frame. Eq. (9) can be simplified as the following shape:

$$
\mathbf{D F}=\mathbf{M}
$$

In this paper, $\mathbf{D}=\left[\begin{array}{c}D_{n} \\ \mathrm{RA} \times \mathrm{D}_{n}\end{array}\right]$ is a matrix of size $6 \times 7, \mathbf{F}$ and $\mathbf{M}=\left[\begin{array}{c}F g-Q \\ M_{t}\end{array}\right]$ are two column vectors with 7 rows. Therefore, Eq. (12) is a redundant equation correlated with Moore-Penrose pseudoinverse. By using the theory of pseudo-inverse, the solution can be derived as:

$$
\mathbf{F}=\mathbf{D}^{+} \mathbf{M}+\left(\mathrm{E}-\mathbf{D}^{+} \mathbf{D}\right) z
$$

where $\mathbf{D}^{+}$is the pseudo-inverse of coefficient matrix D. As shown in Eq. (14), the solution is decomposed into a particular solution $\mathbf{D}^{+} \mathbf{M}$ and a homogeneous solution $\left(E-\mathbf{D}^{+} \mathbf{D}\right) z$, respectively satisfying:

$$
\left\{\begin{array}{c}
\mathbf{D D}^{+} \mathbf{M}=\mathbf{M} \\
\mathbf{D}\left(\mathrm{E}-\mathbf{D}^{+} \mathbf{D}\right) z=\mathbf{0}
\end{array}\right.
$$

The number of solution $\mathbf{F}$ is infinite due to the less number of equations than that of variables. This condition shows up as the infinity of vector $\mathrm{z}$. It affords the potential of optimizing for the actuated forces.

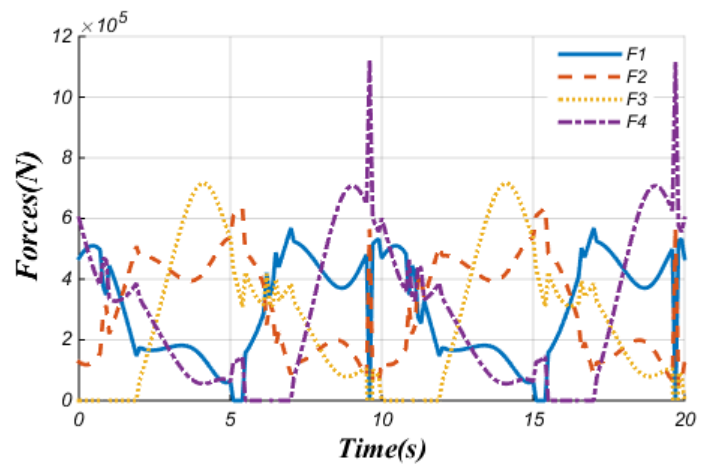

a. The pseudo-inverse solutions

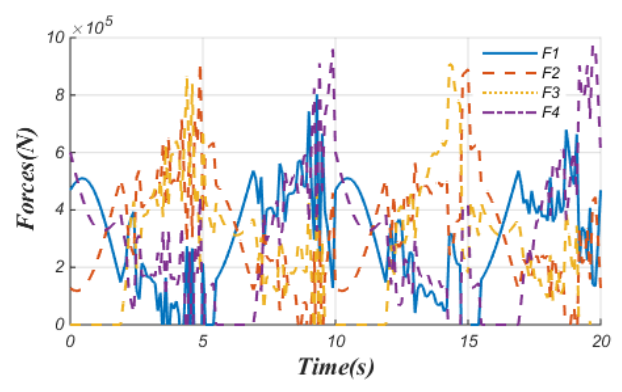

b. The scaling factor solutions

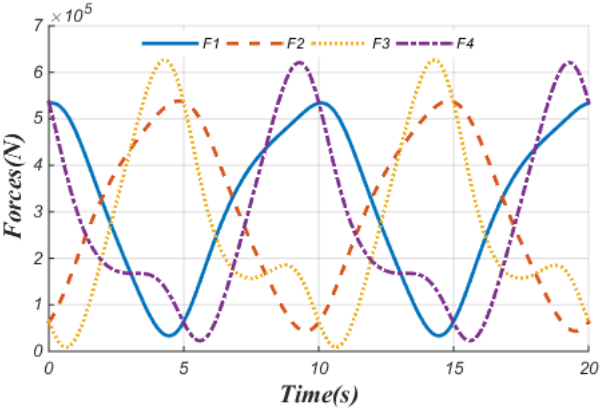

c. The coefficient solutions

Fig.3 The force solutions based on 2-norm of three methods above 


\section{Optimization-based method}

Taking no account of the homogeneous solution, the first formula in Eq. (14) is the pseudo-inverse solution which is aimed at minimizing the 2-norm of the force. And in [16], the pseudo-inverse solutions do not result in any internal forces in redundant parallel manipulators. It is equivalent to seek out the minimization of $\mathbf{F}^{T} \mathbf{F}$,the square of the magnitude of the input forces. In [15], such method cannot find all available output range due to its insufficient to consider input bounds. The $\infty$-norm's method is proposed to settle the question owing to the ability to limit the maximum force using an optimized approach, but it would result in the discontinuity in the solutions.

S.B. Nokleby et al. has presented a method incorporating the actuator limits into the optimization problem by combining the advantages of two method above. It uses a scaling factor named normalized torque vector which can be defined as:

$$
\hat{\tau}=\left\{\hat{\tau}_{1}, \hat{\tau}_{2}, \ldots, \hat{\tau}_{7}\right\}=\left\{\frac{F_{1}}{F_{1 \max }}, \frac{F_{2}}{F_{2 \max }}, \ldots, \frac{F_{7}}{F_{7 \max }}\right\}
$$

Then the problem can be transformed into the optimization problem:

minimize $f(z)=\|\hat{\tau}\|_{p}$

However, in this paper, it is not enough that only consider the actuator limits in order to achieve more even output solutions. In practical situation, the inertia forces resulted from motions of the moving platform is much lower than the gravity since the large mass of loads. Therefore, the forces orientated along the gravity are much more important in the aspect of distributing all actuator outputs. The cosine values of angles $\varphi_{i}$ down from the $\mathrm{z}$ axis are incorporated into a coefficient which is denoted as:

$$
\hat{\mu}=\left\{\hat{\mu}_{1}, \hat{\mu}_{2}, \ldots, \hat{\mu}_{7}\right\}=\left\{\boldsymbol{F}_{1} \cos \varphi_{1}, \boldsymbol{F}_{2} \cos \varphi_{2}, \ldots, \boldsymbol{F}_{7} \cos \varphi_{7}\right\}
$$

In order to find the optimal solution of Eq. ((17), two methods involving norm mentioned above are applied to the optimization approach:

$$
\begin{aligned}
& \operatorname{minimize} f(z)=\|\hat{\mu}\|_{2}=\sqrt{\hat{\mu}_{1}^{2}+\hat{\mu}_{2}^{2}+\cdots+\hat{\mu}_{7}^{2}} \\
& \text { minimize } f(z)=\|\hat{\mu}\|_{\infty}=\max \left(\hat{\mu}_{1}, \ldots, \hat{\mu}_{7}\right)
\end{aligned}
$$

In this method, thanks to the exist of multiplication between $\mathrm{F}$ and the cosine values of $\alpha_{i}$, when the angle is farther from $\mathrm{z}$ axis, the actuator force is much bigger. So that, we can obtain an even solution as well as increase of the integral rigidity offering a large support to the tilted direction on account of uniform distribution of the actuators.

With the given movements as a rotary sinusoidal motion around $\mathrm{X}$ axis of which the amplitude is $5^{\circ}$ and the period is 10s, we can obtain output forces of the prismatic joints as shown in Fig. 3 and Fig. 4 according to the three methods above when the load is about $120 \mathrm{t}$.

In Fig.3, the 2-norm solutions achieved by the pseudo-inverse (denoted as a) and scaling factor (denoted as b) method not only have discontinuous points but also have the inability in distributing the forces proportionally. And in Fig. (3), some forces are reduced to 0. The others get huge outputs in opposite which would cause the singularity. Comparing to $\mathrm{a} \& \mathrm{~b}$, the approach correlated with coefficient $\hat{\mu}$ can solve these problems, with $20 \%$ decline on aspect of the maximal disparity among the actuator forces simultaneously. In order to incorporate the force limits and output range into the problem, the Eq. (19) is used to calculate the forces as Fig.4. However, due to the difference of the $\max (\boldsymbol{F})$ every time, there exists some numerical changes in smooth curves. And in this paper, the changing ranges is not obvious comparing to method c. 


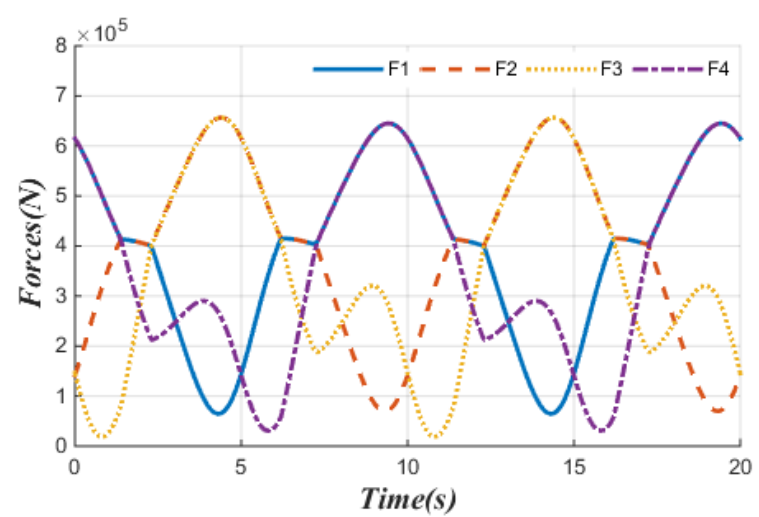

Fig.4 The force solutions based on $\infty$-norm correlated with coefficient

\section{Conclusion}

The paper first introduces a redundant parallel manipulator and give the schematic plot. Then, the motion has been analyzed by the reciprocal screw theory. Next, the inverse kinematic and dynamic problems are solved with the conventional method. Finally, an optimization approach is proposed to solve the pseudo-inverse solution. Through comparison, the approach introduced using coefficient with respect to the dominant direction mainly used in heavy, large loads could solve the redundant problems and get a better-proportioned result. According to this method, we use an optimization approach based on the norms to consider and successfully solve the problem about the discontinuity and the actuator limits.

\section{References}

[1] Pollard, W.L.G., "Spray painting machine," US Patent No. 2,213,108, August26, 1940.

[2] Gough, V.E. and Whitehall, S.G., "Universal tyre test machine," Proceedings of the FISITA Ninth International Technical Congress, pp. 117-137, May, 1962.

[3] Stewart, A Platform with Six Degrees of Freedom. Proc.Instn.Mech.Engrs. (Part I), 1965, 180 (15): 371 386

[4] R. Clavel, "Delta, a fast robot with parallel geometry," in Proc. 18th Int. Symp. Ind. Robots, Lausanne, Switzerland, Apr. 1988, pp. 91-100.

[5] François Pierrot, Optimal Design of a 4-DOF Parallel Manipulator: From Academia to Industry. IEEE TRANSACTIONS ON ROBOTICS, VOL. 25, NO. 2, pp.213-224. APRIL 2009

[6] P. Xu et al. Kinematics analysis of a hybrid manipulator for computer controlled ultra-precision freeform polishing. Robotics and Computer-Integrated Manufacturing 44 (2017) pp.44-56

[7] Z. HUANG. Study on the kinematic characteristics of 3 DOF in-parallel actuated platform mechanism. Mech. Mach. Theory .Vol. 31, No. 8, pp. 999-1007, 1996

[8] A. Hernandez et al. Translational Parallel Manipulator with $\mathrm{Pa}^{2}$ Kinematic Joints. New Advances in Mechanisms, Mechanical Transmissions and Robotics, Mechanisms and Machine Science 46,pp.311-319.2017

[9] Hunt K.H. Kinematic Geometry of Mechanisms. Oxford: Clarendon Press, 1978:3 9

[10] K. Cai et al. Design and control of a 6-degree-of-freedom precision positioning system. Robotics and Computer-Integrated Manufacturing 44. pp.77-96, 2017

[11] Y.F. Zheng, J.Y.S. Luh, Optimal load distribution for two industrial robots handling a single object, in: Proceedings of the 1988 IEEE International Conference on Robotics and Automation, April 24-29, Philadelphia, Pennsylvania, USA, 1988, pp. 344-349.

[12] NAKAMURA and GHODOUSSI. Dynamics Computation of Closed-Link Robot Mechanisms with Nonredundant and Redundant Actuators. IEEE TRANSACTIONS ON ROBOTICS AND AUTOMATION, VOL. 5, NO. 3, June 1989

[13] Mats Isaksson et al. Novel Fault-Tolerance Indices for Redundantly Actuated Parallel Robots. Journal of Mechanical Design. APRIL 2017, Vol. 139 / 042301 
[14] Cha et al. Kinematically-Redundant Variations of the 3-RRR Mechanism and Local Optimization-Based Singularity Avoidance. Mechanics Based Design of Structures and Machines, 35: 15-38, 2007

[15] Travis Baratcart et al. Dynamic Analysis of Continuous Cascaded Generalized Inverse Resolution of Kinematically Redundant Manipulators with Flexible Joints. European Control Conference (ECC) June 24-27, 2014. pp.330-335

[16] S.B. Nokleby et al. Force capabilities of redundantly-actuated parallel manipulators. Mechanism and Machine Theory 40 (2005) 578-599 